\title{
Duodenal Perforation Due to Biliary Stent Migration: Case Report
}

Jalila Ters $^{1 *}$, Oleko Eddy ${ }^{1}$, Wadii Moaquit ${ }^{1}$

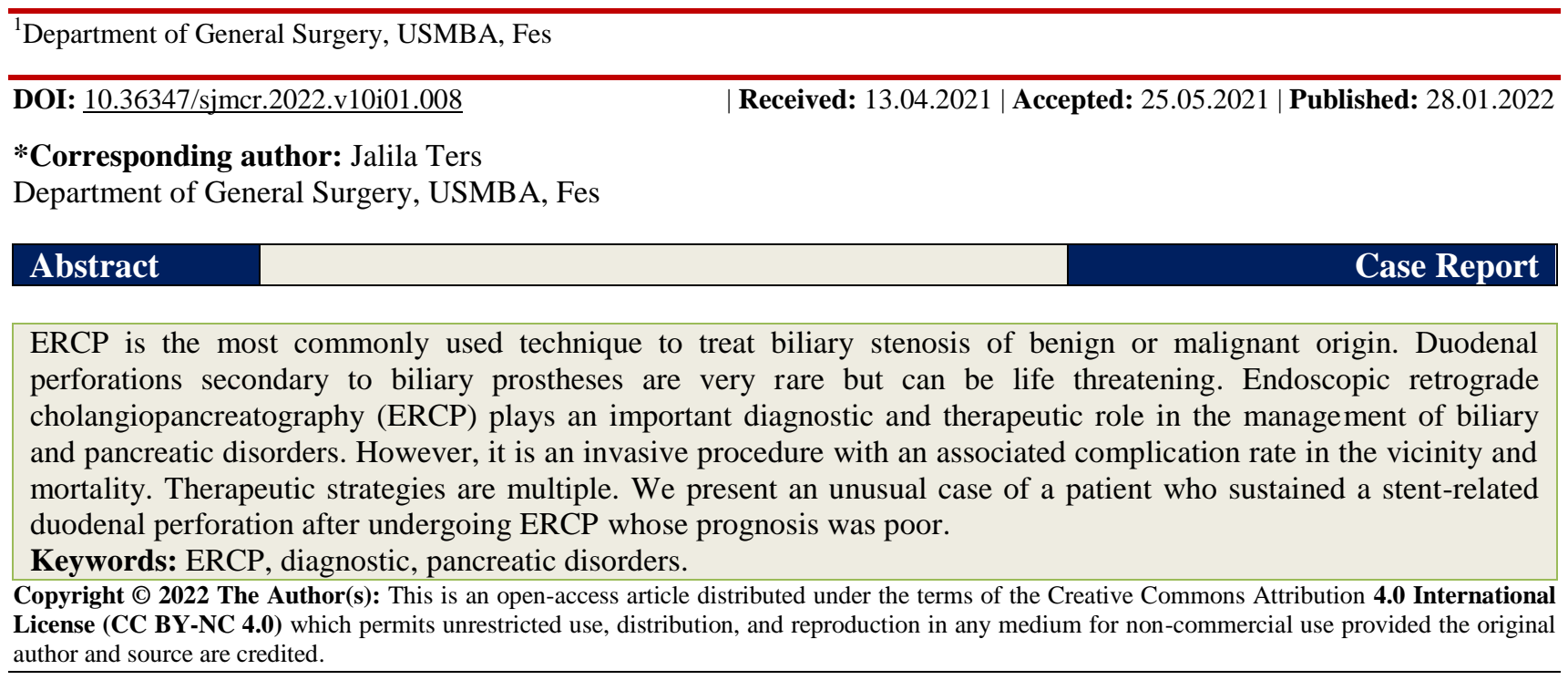

\section{INTRODUCTION}

Endoscopic retrograde cholangiography or ERCP plays a diagnostic and therapeutic role in pancreatobiliary diseases however it has a morbidity rate of $15 \%$ and a mortality of $1 \%$ [1]. In 1979 endoscopic retrograde biliary drainage was used to treat benign or malignant biliary stenosis [1]. The complications of the technique are migration, cholangitis, pancreatitis, hemorrhage and perforation. Among these complications, migration is the most frequent $(10 \%)$, whereas perforation is very rare and represents less than $1 \%$ of complications [2].

It can involve the duodenum, jejunum, ileum, ceacum, colon, and sigmoid colon [1]. Although perforation of the duodenal wall is rare but it remain serious, with a poor prognosis and even early surgical treatment still keeps a high mortality rate [3]. The interval between perforation and surgery is of great importance. The mortality rate increases significantly with late surgical management (>24 h) [4]. Historically duodenal perforations have been treated surgically [5].

However, due to the low incidence of this complication preventive and therapeutic methods continue to be a subject of debate [1]. The aim of this work is to highlight the seriousness of some duodenal perforations due to the late onset of symptoms and which still have a poor prognosis despite emergency surgical treatment.

\section{Case Report}

A 60 years old men with obstructive jaundice due to duodenopancreatic tumor. The biological assessment objectified $\mathrm{Hb}$ 12, WBC 26000, BL 271, BD 232, CRP 43 GOT 347 GPT 267 GGT 1147 PAL 944. The abdominal CT scan was in favor of a voluminous tumor process centered on the duodenopancreatic carrefour locally advanced with dilatation of the intra and extrahepatic bile ducts.

The patient underwent an ERCP which resulted in a stenosing duodenal process with the placement of a $12 * 22 \mathrm{~mm}$ prosthesis. 4 days later the patient presented a peritonitis with generalized abdominal defense and vomiting. The clinical examination revealed a conscious patient tachycardia at $1110 \mathrm{~b} / \mathrm{mn}$ polypneic with generalized abdominal defense.

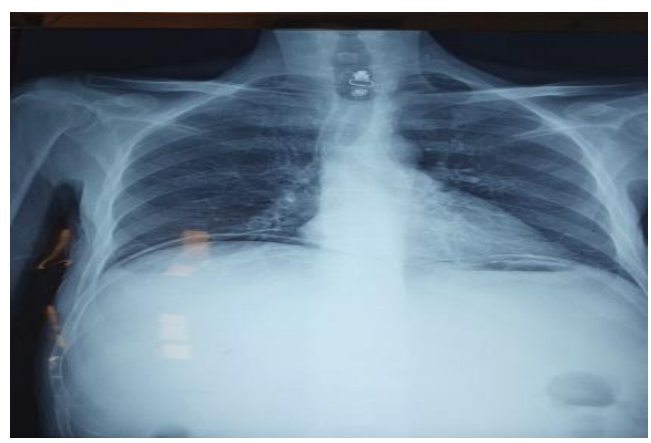

Fig-1: Pneumoperitoneum 
An X-ray centered on the domes showed a pneumoperitoneum.

\section{The biological assessment objectived}

WBC 34000, Hb 7.5; CRP 122; urea 0.71; creatinine 15 .

An emergency abdominal CT scan showed a peri-splenic and peri-renal watery effusion with hydropneumoperitoneum and retro-pneumoperitoneum secondary to a duodenal perforation.

The patient was operated with the realization of a pyloric exclusion of a gastroentero anastomosis and a cholecystotomy an abundant washing and a drainage. The evolution was marked by hemodynamic instability and the patient died 2 days later after non-recovered cardiocirculatory arrest.

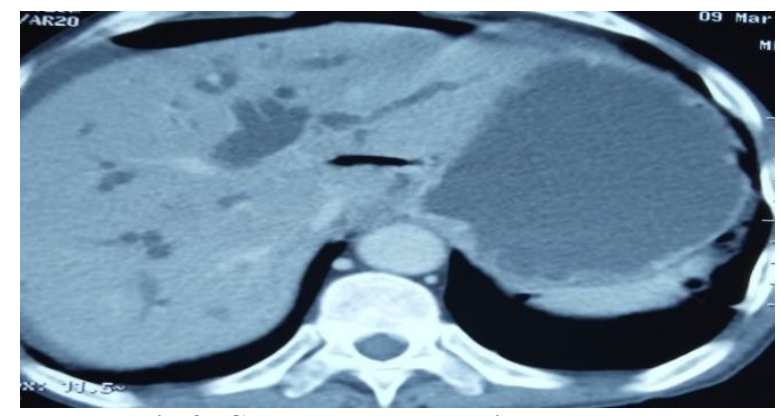

Fig-2: CT scan pneumoperitoneum and retropneumoperitoneum

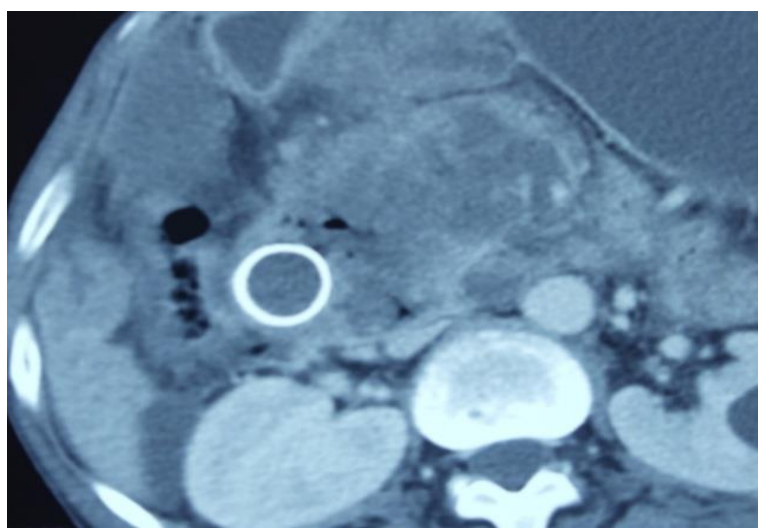

Fig-3: CT scan the stent

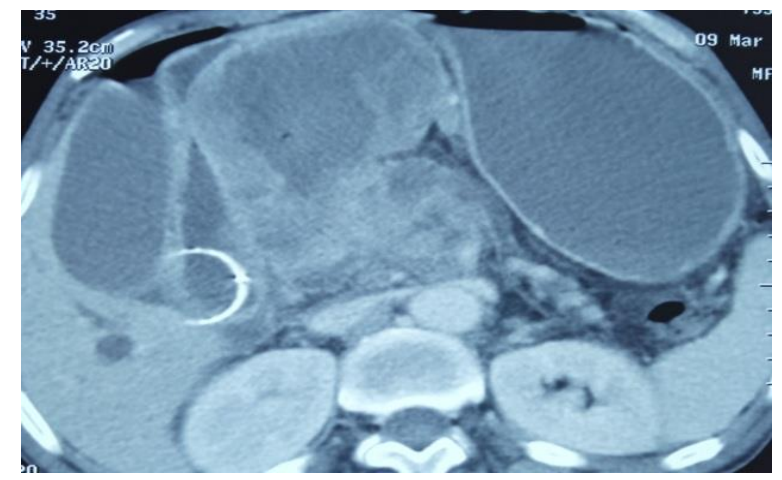

Fig-4: CT scan perisplenic and perirenal hydrperitoneum

\section{DISCUSSION}

Iatrogenic duodenal perforation related to endoscopic retrograde cholangiopancreatography (ERCP) is a very rare and often fatal complication. It is due either to sphincterotomy or to migration of biliary prostheses [6]. Plastic biliary prostheses were first used in 1979 for bile duct drainage. Subsequently, they have become a widely accepted therapy [1].

Several hypotheses have been put forward to explain the migration of the prosthesis causing duodenal perforation, among which are poor insertion technique, choice of prosthesis and inappropriate placement [7].The diagnosis of perforation can be made during ERCP, however, it is often suspected later. By a pneumoperotonium or extravasation of the contrast medium [7].

Clinically the perforation is announced by a fever, abdominal pain or hemodynamic instability. [7] Several investigators have classified post-ERCP ioatrogenic perforation according to the anatomical site and mechanism of perforation $[6,8]$. Stapfer's classification (2000) Howard's classification (1999) and Kim's classification (2011) The Stapfer classification is the most widely used. However, none of the above classifications covers all types of ERCP perforations. [1]. Stapfer et al classified perforations into 4 types in decreasing order of severity $[6,8]$

Type I perforations are located in the lateral or medial wall of the duodenum away from the ampulla of Vater and are usually large and caused by the endoscope itself or the prosthesis with abundant intraand retroperitoneal effusion requiring emergency surgery $[6,8]$.

Type II perforations are perforations of the medial wall of the duodenum; they are perivascular, usually retropenital, and occur during sphincterotomies. These are the most frequent perforations, 15 to $68 \%$ of cases [8]; they are treated by conservative or minimally invasive therapies [6].

Type III are distal perforations. They are small and are treated by conservative management.

Type IV are tiny retroperitoneal perforations that can be managed conservatively.

According to the classification of Stapfer et al, our patient's perforation can be classified as a type I perforation, which is due to the prosthesis with a peritoneal effusion. There is no clear consensus for primary repair of iatrogenic perforations due to ERCP because of the limited number of cases [6]. Treatment depends on the mechanism of the perforation, the site of the degree of effusion, and the patient's condition [9]. Perforations (type I) can be treated endoscopically with fibrin sealant or endoclips; surgery is recommended if 
there is an intraperitoneal or retroperitoneal effusion,massive subcutaneous emphysema or a large perforation $[6,9]$.

For duodenal perforations secondary to biliary prostheses, the treatment of choice is endoscopic removal of the prosthesis, followed by closure of the perforation with clips or glue. Surgery should be reserved for patients with peritonitis [6], which is the case for our patient.

For other types of perforation, treatment is conservative $[6,9]$.

\section{Conclusion}

According to the literature, although nonsurgical suture therapies are not yet widely accepted as primary treatment for duodenal perforation related to ERCP and biliary prostheses, endoscopic treatment may replace surgical management in the future.

However, for successful management, prompt recognition and early management, including endoscopic closure or surgery, are the main determinants to decrease morbidity and mortality, and early diagnosis of perforation remains the key requirement for optimal outcome.

In the case of our patient, the delay in diagnosis, the size of the perforation and the type of perforation all contributed to the therapeutic failure.

\section{REFERENCES}

1. Wang X, Qu J, Li K. Duodenal perforations secondary to a migrated biliary plastic stent successfully treated by endoscope: case-report and review of the literature. BMC Gastroenterol. 12 mai 2020;20(1):149.

2. Angiò LG, Sfuncia $G$, Viggiani $P$, Faro $G$, Bonsignore A, Licursi $\mathrm{M}$, et al. [Management of perforations as adverse events of ERCP plus ES. Personal experience]. G Chir. déc 2009;30(11-12):520-30.

3. Wu X, Li G, Liu Z. A rare case of duodenum perforation after biliary stenting under endoscopic retrograde cholangiopancreatography: a case report. Ann Transl Med. déc 2020;8(24):1690.

4. Avgerinos DV, Llaguna $\mathrm{OH}$, Lo AY, Voli J, Leitman IM. Management of endoscopic retrograde cholangiopancreatography: related duodenal perforations. Surg Endosc. avr 2009;23(4):833-8.

5. Machado NO. Management of duodenal perforation post-endoscopic retrograde cholangiopancreatography. When and whom to operate and what factors determine the outcome? A review article. JOP. 10 janv 2012;13(1):18-25.

6. Lee TH, Han J-H, Park S-H. Endoscopic Treatments of Endoscopic Retrograde Cholangiopancreatography-Related Duodenal Perforations. Clin Endosc. sept 2013;46(5):522-8.

7. Lo $\mathrm{CH}$, Chung $\mathrm{S}$, Bohmer RD. A devastating complication: duodenal perforation due to biliary stent migration. Surg Laparosc Endosc Percutan Tech. déc 2008;18(6):608-10.

8. Theopistos V, Theocharis G, Konstantakis C, Kitrou P, Kehagias I, Triantos C, et al. NonOperative Management of Type 2 ERCP-Related Retroperitoneal Duodenal Perforations: A 9-Year Experience From a Single Center. Gastroenterology Res. juin 2018;11(3):207-12.

9. Vezakis A, Fragulidis G, Polydorou A. Endoscopic retrograde cholangiopancreatography-related perforations: Diagnosis and management. World J Gastrointest Endosc. 10 oct 2015;7(14):1135-41. 